\title{
Metrical common fixed point theorems without completeness and closedness
}

\author{
Dhananjay Gopal ${ }^{1 *}$, Mohammad Imdad ${ }^{2}$ and Mujahid $\mathrm{Abbas}^{3}$
}

\author{
* Correspondence: \\ gopaldhananjay@yahoo.in \\ 'Department of Mathematics and \\ Humanities, S.V. National Institute \\ of Technology, Surat, Gujarat, India \\ Full list of author information is \\ available at the end of the article
}

\begin{abstract}
In this article, we point out that some recent results proved in Babu and Alemayehu are corollaries of the main result of an article due to Ali and Imdad. However, combining the notions of $R$-weakly commuting property of type ( $\mathrm{Ag}$ ) (due to Pathak et al. together with sequential continuity of type $(\mathrm{Ag})$ (to be introduced in this note) (also alternately $R$-weakly commuting property of type (Af) (due to Pathak et al.) together with sequential continuity of type (Af) (to be introduced in this note)) these results are improved which are possibly new results in the literature of metric fixed point theory.

Mathematics Subject Classification (2000): 47H06; 47H10.

Keywords: $R$-weakly commuting pair of maps of type $(\mathrm{Ag}), R$-weakly commuting pair of maps of type $(A f)$, sequentially continuity of type $(A g)$, sequentially continuity of type $(A f)$, the property (E.A); weakly compatible maps, occasionally weakly compatible maps
\end{abstract}

\section{Introduction and preliminaries}

Recently, Babu and Alemayehu [1] proved the following results as improvements over the relevant ones presented in Kameswari [2].

Proposition 1.1. [1] Let $A, B, S$ and $T$ be four self maps of a metric space $(X, d)$ satisfying the inequality

$$
\begin{aligned}
& {[d(A x, B y)]^{2} \leq c_{1} \max \left\{[d(S x, A x)]^{2},[d(T y, B y)]^{2},[d(S x, T y)]^{2}\right\}} \\
& +c_{2} \max \{d(S x, A x) d(S x, B y), d(T y, A x) d(T y, B y)\}+c_{3} d(T y, A x) d(S x, B y)
\end{aligned}
$$

for all $x, y \in X$ where $c_{1}, c_{2}, c_{3} \geq 0$ and $c_{1}+c_{3}<1$. If either

(i) $B(X) \subseteq S(X)$, the pair $(B, T)$ satisfies the property (E.A.) and $T(X)$ is a closed subset of $X$, or

(ii) $A(X) \subseteq T(X)$, the pair $(A, S)$ satisfies the property (E.A.) and $S(X)$ is a closed subset of $X$,

then the pairs $(A, S)$ and $(B, T)$ have a coincidence point each.

Theorem 1.1. [1] If, in addition to the hypotheses of Proposition 1.1, both the pairs $(A, S)$ and $(B, T)$ are occasionally weakly compatible, then the maps $A, B, S$ and $T$ have a unique common fixed point in $X$.

(c) 2012 Gopal et al.; licensee Springer. This is an open access article distributed under the terms of the Creative Commons Attribution License (http://creativecommons.org/licenses/by/2.0), which permits unrestricted use, distribution, and reproduction in any medium, provided the original work is properly cited. 
Proposition 1.2. [1] Let $A, B, S$ and $T$ be four self-maps of a metric space $(X, d)$ satisfying the inequality (1.1) (of Proposition (1.1)). If the pairs $(A, S)$ and $(B, T)$ share the common property (E.A.) and $S(X)$ as well as $T(X)$ are closed subsets of $X$, then the pairs $(A, S)$ and $(B, T)$ have a coincidence point each.

Theorem 1.2. [1] If, in addition to the hypotheses of Proposition 1.2, both the pairs $(A, S)$ and $(B, T)$ are occasionally weakly compatible, then the maps $A, B, S$ and $T$ have a unique common fixed point in $X$.

First, we point out that preceding results can be deduced as corollaries of Theorem 3.1 due to Ali and Imdad [3] which runs as follows.

Theorem 1.3. [3] Let $A, B, S$ and $T$ be four self maps of a metric space $(X, d)$ satisfying the inequality

$$
F(d(A x, B y), d(S x, T y), d(A x, S x), d(B y, T y), d(S x, B y), d(T y, A x)) \leq 0
$$

for all $x, y \in X$ and $F \in \Psi$, where $\Psi$ is the class of lower semi-continuous (in short 1 . s.c.) functions $F: \mathbb{R}_{+}^{6} \rightarrow \mathbb{R}$ enjoying the following properties:

$F_{1} F(t, 0, t, 0,0, t)>0$, for all $t>0$;

$F_{2} F(t, 0,0, t, t, 0)>0$, for all $t>0$;

$F_{3} F(t, t, 0,0, t, t)>0$, for all $\mathrm{t}>0$.

Suppose that

(i) the pairs $(A, S)$ and $(B, T)$ share the common property (E.A.),

(ii) $S(X)$ and $T(X)$ are closed subsets of $X$.

Then the pairs $(A, S)$ and $(B, T)$ have a coincidence point each. Moreover, $A, B, S$, and $T$ have a unique common fixed point provided both the pairs $(A, S)$ and $(B, T)$ are weakly compatible.

Recently, Gopal et al. [4] extended Theorem 1.3 to symmetric spaces involving a sequence of mappings in symmetric spaces. In order to state this result, we need some terminology which can be summarize as follows:

A symmetric $d$ in respect of a non-empty set $X$ is a function $d: X \times X \rightarrow[0, \infty)$ which satisfies $d(x, y)=d(y, x)$ and $d(x, y)=0 \Leftrightarrow x=y$ (for all $x, y \in X)$. If $d$ is a symmetric on a set $X$, then for $x \in X$ and $\epsilon>0$, we write $B(x, \epsilon)=\{y \in X: d(x, y)<\epsilon\}$. A topology $\tau(d)$ on $X$ is given by the sets $U$ (along with empty set) in which for each $x \in$ $U$, one can find some $\epsilon>0$ such that $B(x, \epsilon) \subset U$. A set $S \subset X$ is a neighborhood of $x$ $\in X$ if and only if there is a $U$ containing $x$ such that $x \in U \subset S$. A symmetric $d$ is said to be a semi-metric if for each $x \in X$ and for each $\epsilon>0, B(x, \epsilon)$ is a neighborhood of $x$ in the topology $\tau(d)$. Thus a symmetric (resp., a semi-metric) space $X$ is a topological space whose topology $\tau(d)$ on $X$ is induced by a symmetric (resp., a semi-metric) $d$. Notice that $\lim _{n \rightarrow \infty} d\left(x_{n}, x\right)=0$ if and only if $x_{n} \rightarrow x$ in the topology $\tau(d)$. The distinction between a symmetric and a semi-metric is apparent as one can easily construct a symmetric $d$ such that $B(x, \epsilon)$ need not be a neighborhood of $x$ in $\tau(d)$. As symmetric spaces are not essentially Hausdorff and the symmetric $d$ is also not continuous in general, therefore in order to prove fixed point theorems some additional axioms are required. 
The following axioms are relevant to this note which are available in Aliouche [5], Galvin and Shore [6], Hicks and Rhoades [7], and Wilson [8]. From now on symmetric as well as semi-metric spaces will be denoted by $(X, d)$.

$\left(W_{3}\right)$ : (cf. [8]) Given $\left\{x_{n}\right\}, x$ and $y$ in $X$ with $d\left(x_{n}, x\right) \rightarrow 0$ and $d\left(x_{n}, y\right) \rightarrow 0$ imply $x=$ y.

(1C): (cf. [9]) A symmetric $d$ is said to be 1-continuous if $\lim _{n \rightarrow \infty} d\left(x_{n}, x\right)=0$ implies $\lim _{n \rightarrow \infty} d\left(x_{n}, y\right)=d(x, y)$.

(HE): (cf. [5]) Given $\left\{x_{n}\right\},\left\{y_{n}\right\}$ and an $x$ in $X$ with $d\left(x_{n}, x\right) \rightarrow 0$ and $d\left(y_{n}, x\right) \rightarrow 0$ imply $d\left(x_{n}, y_{n}\right) \rightarrow 0$.

Now we are equipped to state the following theorem.

Theorem 1.4. (cf. [4]) Let $(X, d)$ be a symmetric space satisfying $(1 C)$ and $(H E)$. Let $S, T, A_{k}$, for $k=1,2, \ldots$, be self mappings of $X$ satisfying the inequality

$$
\phi\left(d\left(A_{1} x, A_{k} y\right), d(S x, T y), d\left(S x, A_{1} x\right), d\left(T y, A_{k} y\right), d\left(S x, A_{k} y\right), d\left(T y, A_{1} x\right)\right) \leq 0,
$$

for all $x, y \in X$ and $\varphi \in \Psi$. Suppose that the pairs $\left(A_{1}, S\right)$ and $\left(A_{k}, T\right)$ for $k>1$ share the common property (E.A.), $S(X)$ and $T(X)$ are closed subsets of $X$. Then the pairs $\left(A_{1}, S\right)$ and $\left(A_{k}, T\right)$ have a coincidence point. Moreover $S, T$ and $A_{k}$ have a unique common fixed point provided both the pairs $\left(A_{1}, S\right)$ and $\left(A_{k}, T\right)$ for each $k>1$ are weakly compatible.

In the event of single valued pair of maps, the notion of occasional weak compatibility reduces to weak compatibility due to unique coincidence point of the underlying maps (see [10]) which is always ensured by underlying contraction condition. Hence, weak compatibility remains the minimal commutativity condition for the existence of common fixed point for contractive type mappings.

In fact, it was claimed by some authors (e.g., $[11,12]$ ) that the notion of occasional weak compatibility relaxes the requirement of completeness as well as closedness condition on underlying space or subspaces in proving common fixed point theorems for contractive type mappings.

Now, there arises a natural question: "which optimal class of mappings will do the job?" The present article is an attempt to give an affirmative answer of the above question. In this perspective, we utilize the combined idea of $R$-weakly commuting of type $(A g)$ due to Pathak et al. [13] together with sequentially continuity of type $(A g)$ (also alternately $R$-weakly commuting of type $(A f)$ due to Pathak et al. [13] together with sequentially continuity of type $(A f))$.

Before presenting our main results, we recall the relevant definitions and results needed in our latter discussion. For details, we refer to $[1,3,13,14]$ and references mentioned therein.

Definition 1.1. A pair $(f, g)$ of self maps defined on a metric space $(X, d)$ is said to be:

(i) compatible if $\lim _{n \rightarrow \infty} d\left(f g x_{n}, g f x_{n}\right)=0$, whenever $\left\{x_{n}\right\}$ is a sequence in $X$ such that $\lim _{n \rightarrow \infty} f x_{n}=\lim _{n \rightarrow \infty} g x_{n}=t$ for some $t \in X$, 
(ii) non-compatible if there exists some sequence $\left\{x_{n}\right\}$ in $X$ such that $\lim _{n \rightarrow \infty} f x_{n}=\lim _{n \rightarrow \infty} g x_{n}=t$ for some $t \in X$ but $\lim _{n \rightarrow \infty} d\left(f g x_{n}, g f x_{n}\right)$ is either non-zero or non-existent,

(iii) $R$-weakly commuting of type $(A g)$ on $X$ if $d(f f x, g f x) \leq R d(f x, g x)$ for some $R>0$, where $x$ varies over $X$,

(iv) $R$-weakly commuting of type (Af) on $X$ if $d(f g x, g g x) \leq R d(f x, g x)$ for some $R>$ 0 , where $x$ varies over $X$,

(v) weakly compatible (or partially commuting or coincidentally commuting) if $f$ and $g$ commute on the set of coincidence points, that is, if $f x=g x$ for some $x$ in $X$ implies that $f g x=g f x$,

(vi) occasionally weakly compatible iff there is a point $x$ in $X$ which is a coincidence point of $f$ and $g$ at which $f$ and $g$ commutes, i.e., there exist a point $x$ in $X$ such that $f x=g x$ and $f g x=g f x$,

(vii) sub-compatible iff there exists a sequence $\left\{x_{n}\right\}$ in $X$ such that $\lim _{n \rightarrow \infty} f x_{n}=\lim _{n \rightarrow \infty} g x_{n}=t$ for some $t \in X$ and which satisfy $\lim _{n \rightarrow \infty} d\left(f g x_{n}, g f x_{n}\right)=0$,

(viii) tangential (or satisfying the property (E.A.)) if there exists a sequence $\left\{x_{n}\right\}$ in $X$ such that $\lim _{n \rightarrow \infty} f x_{n}=\lim _{n \rightarrow \infty} g x_{n}=t$ for some $t \in X$,

(ix) reciprocally continuous if $\lim _{n \rightarrow \infty} f g x_{n}=f t$ and $\lim _{n \rightarrow \infty} g f x_{n}=g t$ whenever $\left\{x_{n}\right\}$ is a sequence in $X$ such that $\lim _{n \rightarrow \infty} f x_{n}=\lim _{n \rightarrow \infty} g x_{n}=t$ for some $t \in X$,

(x) sub-sequential continuous iff there exists a sequence $\left\{x_{n}\right\}$ in $X$ such that $\lim _{n \rightarrow \infty}$, $f x_{n}=\lim _{n \rightarrow \infty} g x_{n}=t$ for some $t \in X$ and which satisfy $\lim _{n \rightarrow \infty} f g x_{n}=f t$ and $\lim _{n \rightarrow \infty} g f x_{n}=g t$.

For further information on weak commutativity conditions, one is also referred $[15,16]$. Notice that (iii) and (iv) $\Rightarrow(v) \Rightarrow($ vi $) \Rightarrow$ (vii) but the converse implications are not true. Let us agree to call a pair $(f, g)$ of maps to be nonvacuously reciprocally continuous if there exists at least one sequence meeting the requirement of the definition of reciprocal continuity. Otherwise, the pair of maps $(f, g)$ may be termed as vacuously reciprocally continuous. Notice that every nonvacuously reciprocally continuous pair of maps $(f, g)$ is naturally sub-sequentially continuous. However, there do exist subsequentially continuous pairs of maps which are neither continuous nor reciprocally continuous [14].

Before proving our results, it can be pointed out that earlier stated results of Babu and Alemayehu [1] can be deduced from Theorem 1.3 as inequality (1.1) falls in the format of implicit relation utilized in Ali and Imdad [3] while the notions of weak compatibility and occasional weak compatibility coincide in the presence of contraction conditions. One may also notice that improved versions of earlier stated results can also be deduced from Theorem 1.4 contained in [4].

\section{Main results}

Motivated by the authors of $[13,14,17]$, we introduce the following definitions.

Definition 2.1. A pair $(f, g)$ of self maps defined on a metric space $(X, d)$ is said to be sequentially continuous of type $(A g)$ iff there exists a sequence $\left\{x_{n}\right\}$ in $X$ such that 
$\lim _{n \rightarrow \infty} g f x_{n}=g t$ for some $t \in X$ and $\lim _{n \rightarrow \infty} f f x_{n}=f t$ and $\lim _{n \rightarrow \infty} g f x_{n}=g t$.

Definition 2.2. A pair $(f, g)$ of self maps defined on a metric space $(X, d)$ is said to be sequentially continuous of type (Af) iff there exists a sequence $\left\{x_{n}\right\}$ in $X$ such that $\lim _{n \rightarrow \infty} f x_{n}=\lim _{n \rightarrow \infty} g x_{n}=t$ for some $t \in X$ and $\lim _{n \rightarrow \infty} f g x_{n}=f t$ and $\lim _{n \rightarrow \infty} g g x_{n}=g t$.

Notice that the notions of sub-sequential continuity and sequentially continuity of type $(A g)$ (also alternately notions of sub-sequential continuity and sequentially continuous of type $(A f)$ ) are independent of each other. To substantiate this view point, we furnish the following examples:

Example 2.1. Consider $X=[0, \infty)$ endowed with the natural metric $d$ and define $f, g$ : $X \rightarrow X$ by

$$
\begin{aligned}
& f(x)=\left\{\begin{array}{ll}
0 & \text { if } x=0, \\
1+x & \text { if } x \in(0,1], \\
2 x-1 & \text { if } x \in(1, \infty)
\end{array}\right. \text { and } \\
& g(x)= \begin{cases}1-x & \text { if } x \in[0,1), \\
3 x-2 & \text { if } x \in[1, \infty) .\end{cases}
\end{aligned}
$$

If we choose $x_{n}=\frac{1}{n}$ for $n=1,2, \ldots$, then

$$
\begin{aligned}
& \lim _{n \rightarrow \infty} f\left(x_{n}\right)=\lim _{n \rightarrow \infty}\left(1+\frac{1}{n}\right)=1, \\
& \lim _{n \rightarrow \infty} g\left(x_{n}\right)=\lim _{n \rightarrow \infty}\left(1+\frac{1}{n}\right)=1 \\
& \lim _{n \rightarrow \infty} f g\left(x_{n}\right)=\lim _{n \rightarrow \infty} f\left(1-\frac{1}{n}\right)=\lim _{n \rightarrow \infty}\left(2+\frac{1}{n}\right)=2=f(1), \\
& \lim _{n \rightarrow \infty} g f\left(x_{n}\right)=\lim _{n \rightarrow \infty} g\left(1+\frac{1}{n}\right)=\lim _{n \rightarrow \infty}\left(1+\frac{3}{n}\right)=1=g(1), \text { and } \\
& \lim _{n \rightarrow \infty} f f\left(x_{n}\right)=\lim _{n \rightarrow \infty} f\left(1+\frac{1}{n}\right)=\lim _{n \rightarrow \infty}\left(2\left(1+\frac{1}{n}\right)-1\right)=1 \neq 2=f(1) .
\end{aligned}
$$

Thus, the pair $(f, g)$ is subsequentially continuous but not sequentially continuous of type (Ag).

Example 2.2. Consider $X=[2,20]$ endowed with the natural metric $d$ and define $f, g$ : $X \rightarrow X$ by

$$
\begin{aligned}
& f x=\left\{\begin{array}{l}
2, \text { if } x=2, \quad \text { or } \quad x>5 \\
6, \text { if } 2<x \leq 5
\end{array}\right. \\
& g x= \begin{cases}10, & \text { if } x=2 \\
12, & \text { if } 2<x \leq 5 \\
\frac{x+1}{3}, & \text { if } x>5\end{cases}
\end{aligned}
$$

Here, the pair $(f, g)$ is sequentially continuous of type $(A g)$ but not subsequently continuous. To substantiate the claim, one can choose $\left\{x_{n}=\left(5+\frac{1}{n}\right)\right\}$ for $n=1,2, \ldots$ in $X$.

Now, we prove our results on common fixed point of four maps which not only improve the results contained in [1] but possibly give rise new results. 
Theorem 2.1. Let $A, B, S$, and $T$ be four self maps of a metric space $(X, d)$. If the pairs $(A, S)$ and $(B, T)$ are sequentially continuous of type (Ag) as well as $R$-weakly commuting of type $(A g)$, then pairs $(A, S)$ and $(B, T)$ have a coincidence point. If pairs $(A, S)$ and $(B, T)$ satisfy the following inequality

$$
F(d(A x, B y), d(S x, T y), d(A x, S x), d(B y, T y), d(S x, B y), d(T y, A x)) \leq 0
$$

for all $x, y \in X$ and $F \in \Psi$, where $\Psi=\left\{F \mid F: \mathbb{R}_{+}^{6} \rightarrow \mathbb{R}\right.$ is lower semi-continuous functions (l.s.c.) with $F(t, t, 0,0, t, t)>0$, for all $t>0\}$, then $A, B, S$, and $T$ have a unique common fixed point.

Proof. Since the pair $(A, S)$ is sequentially continuous of type $(A g)$ as well as $R$-weakly commuting of type $(A g)$, there exists a sequence $\left\{x_{n}\right\}$ in $X$ with $\lim _{n \rightarrow \infty} A x_{n}=\lim _{n \rightarrow \infty} S x_{n}=u$ (for some $u \in X$ ) such that $\lim _{n \rightarrow \infty} A A x_{n}=A u$ and $\lim _{n \rightarrow \infty} S A x_{n}=S u$. Also, $\lim _{n \rightarrow \infty} d\left(A A x_{n}, S A x_{n}\right) \leq R \lim _{n \rightarrow \infty} d\left(A x_{n}, S x_{n}\right)=0$ so that $d(A u, S u)=\lim _{n \rightarrow \infty} d\left(A A x_{n}, S A x_{n}\right)=0$, i.e., $u$ is a coincidence point of the pair $(A, S)$.

Similarly, in respect of pair $(B, T)$, there also exists a sequence $\left\{y_{n}\right\}$ with $\lim _{n \rightarrow \infty} B y_{n}=\lim _{n \rightarrow \infty} T y_{n}=v \quad\left(\right.$ for some $\quad v \in X$ ) such that $\lim _{n \rightarrow \infty} B B y_{n}=B v$ and $\lim _{n \rightarrow \infty} T B y_{n}=T v$. Also, $\quad \lim _{n \rightarrow \infty} d\left(B B y_{n}, T B y_{n}\right) \leq R \lim _{n \rightarrow \infty} d\left(B y_{n}, T y_{n}\right)=0$, so that $d(B v, T v)=\lim _{n \rightarrow \infty} d\left(B B y_{n}, T B y_{n}\right)=0$, i.e., $v$ is a coincidence point of the pair $(B, T)$.

Now, we show that $u=v$. If it is not so, then using inequality (2.1), we have

$$
F\left(d\left(A x_{n}, B y_{n}\right), d\left(S x_{n}, T y_{n}\right), d\left(A x_{n}, S x_{n}\right), d\left(B y_{n}, T y_{n}\right), d\left(S x_{n}, B y_{n}\right), d\left(T y_{n}, A x_{n}\right)\right) \leq 0 .
$$

Taking the limit as $n \rightarrow \infty$ and using the l.s.c. of $F$, we obtain

$$
F(d(u, v), d(u, v), 0,0, d(u, v), d(u, v)) \leq 0,
$$

a contradiction so that $u=v$.

Next, we assert that $A u=u$. Let on contrary that $A u \neq u$. On using (2.1), we get

$$
F\left(d\left(A u, A y_{n}\right), d\left(S u, T y_{n}\right), d(A u, S u), d\left(B y_{n}, T y_{n}\right), d\left(S u, B y_{n}\right), d\left(T y_{n}, A u\right)\right) \leq 0 .
$$

Taking the limit as $n \rightarrow \infty$ and using the l.s.c. of $F$, we get

$$
F(d(A u, u), d(A u, u), 0,0, d(A u, u), d(u, A u)) \leq 0,
$$

a contradiction. Hence $u=A u=S u$. To prove $B u=u$, assume on contrary that $B u \neq$ $u$. Using (2.1), we obtain

$$
F(d(A u, B u), d(S u, T u), d(A u, S u), d(B u, T u), d(S u, B u), d(T u, A u)) \leq 0,
$$

or

$$
F(d(u, B u), d(u, B u), 0,0, d(u, B u), d(B u, u)) \leq 0,
$$

a contradiction. Thus $u$ is a common fixed point of $A, B, S$ and $T$.

Finally, suppose that there exists another common fixed point $z$ of $A, B, S$ and $T$ such that $z \neq u$. Then, on using inequality (2.1), we have

$$
F(d(A u, B z), d(S u, T z), d(A u, S u), d(B z, T z), d(S u, B z), d(T z, A u)) \leq 0,
$$


or

$$
F(d(u, z), d(u, z), 0,0, d(u, z), d(u, z)) \leq 0,
$$

which is a contradiction. Hence $u=z$. Thus, $u$ is the unique common fixed point of $A, B, S$, and $T$. This completes the proof.

Restricting Theorem 2.1 to a pair of mappings, we deduce the following:

Corollary 2.1. Let $A$ and $S$ be two self maps of a metric space $(X, d)$. If the pair $(A$, $S)$ is sequentially continuous of type $(A g)$ as well as $R$-weakly commuting of type ( $A g)$, then pair $(A, S)$ has a coincidence point. If pair $(A, S)$ satisfies the inequality

$$
F(d(A x, A y), d(S x, S y), d(A x, S x), d(A y, S y), d(S x, A y), d(S y, A x)) \leq 0
$$

for all $x, y \in X$ and $F \in \Psi$, where $\Psi=\left\{F \mid F: \mathbb{R}_{+}^{6} \rightarrow \mathbb{R}\right.$ is lower semi-continuous functions (1.s.c.) and $F(t, t, 0,0, t, t)>0$, for all $t>0\}$, then $A$ and $S$ have a unique common fixed point.

Remark 2.1. A careful examination of the proof reveals the fact that Theorem 2.1 (hence also Corollary 2.1) remains valid in symmetric space $(X, d)$ wherein $d$ is continuous.

Alternately, using $R$-weakly commuting property of type $(A f)$ (due to Pathak et al. [13]) together with sequential continuity of type $(A f)$, we have the following:

Theorem 2.2. Let $A, B, S$, and $T$ be four self maps of a metric space $(X, d)$. If the pairs $(A, S)$ and $(B, T)$ are sequentially continuous of type $(A f)$ as well as $R$-weakly commuting of type $(A f)$, then pairs $(A, S)$ and $(B, T)$ have a coincidence point. If pairs $(A, S)$ and $(B, T)$ satisfy the inequality (2.1) (of Theorem 2.1), then $A, B, S$ and $T$ have a unique common fixed point.

Proof. Since the pair $(A, S)$ is sequentially continuous of type $(A f)$ and $R$-weakly commuting of type $(A f)$, there exist sequence $\left\{x_{n}\right\}$ in $X$ with $\lim _{n \rightarrow \infty} A x_{n}=\lim _{n \rightarrow \infty} S x_{n}=u$, for some $u \in X$ such that $\lim _{n \rightarrow \infty} A S x_{n}=A u$ and $\lim _{n \rightarrow \infty} S S x_{n}=S u$. Also, $\lim _{n \rightarrow \infty} d\left(A S x_{n}, S S x_{n}\right)=0$. Thus, we get $d(A u, S u)=\lim _{n \rightarrow \infty} d\left(A S x_{n}, S S x_{n}\right)=0$, i.e., $u$ is a coincidence point of the pair $(A, S)$.

Similarly, in respect of pair $(B, T)$, there also exists a sequence $\left\{y_{n}\right\}$ in $X$ with $\lim _{n \rightarrow \infty} B y_{n}=\lim _{n \rightarrow \infty} T y_{n}=v$, for some $v \in X$ such that $\lim _{n \rightarrow \infty} B T y_{n}=B v$ and $\lim _{n \rightarrow \infty} T T y_{n}=T v$. Also, $\quad \lim _{n \rightarrow \infty} d\left(B T y_{n}, T T y_{n}\right) \leq R \lim _{n \rightarrow \infty} d\left(B y_{n}, T y_{n}\right)=0 . \quad$ Thus, we $d(B v, T v)=\lim _{n \rightarrow \infty} d\left(B B y_{n}, T B y_{n}\right)=0$, i.e., $v$ is a coincidence point of the pair $(B, T)$. The rest of the proof can be completed on the lines of above Theorem 2.1. This concludes the proof.

Restricting Theorem 2.2 to a pair of mappings, we deduce the following:

Corollary 2.2. Let $A$ and $S$ be two self maps of a metric space $(X, d)$. If the pair $(A$, $S$ ) is sequentially continuous of type $(A f)$ as well as $R$-weakly commuting of type ( $A f)$, then the pair $(A, S)$ has a coincidence point. If pair $(A, S)$ satisfies the inequality

$$
F(d(A x, A y), d(S x, S y), d(A x, S x), d(A y, S y), d(S x, A y), d(S y, A x)) \leq 0
$$


for all $x, y \in X$ and $F \in \Psi$, where $\Psi=\left\{F \mid F: \mathbb{R}_{+}^{6} \rightarrow \mathbb{R}\right.$ is lower semi-continuous functions (l.s.c.) and $F(t, t, 0,0, t, t)>0$, for all $t>0\}$, then $A$ and $S$ have a unique common fixed point.

Remark 2.2. A careful examination of the proof reveals the fact that Theorem 2.2 (hence also Corollary 2.2) remains valid in symmetric space $(X, d)$ wherein $d$ is continuous.

Finally, we present an example to demonstrate the validity of the hypotheses and degree of generality of our results over comparable ones from the existing literature.

Example 2.3. Consider $X=[2,20)$ endowed with the natural metric $d$ and define $A$, $S: X \rightarrow X$ by

$$
\begin{aligned}
& A x=\left\{\begin{array}{l}
2, \quad \text { if } \quad x=2, \quad \text { or } \quad x>5, \\
\frac{4}{3} x, \quad \text { if } \quad 2<x \leq 5,
\end{array}\right. \\
& S x= \begin{cases}2, & \text { if } \quad x=2 \\
18, & \text { if } 2<x \leq 5 \\
\frac{x+1}{3}, & \text { if } \quad x>5 .\end{cases}
\end{aligned}
$$

In respect of the sequence $x_{n}=\left(5+\frac{1}{n}\right)$ for $n=1,2, \ldots$ in $X$, we have

$$
\begin{aligned}
& \lim _{n \rightarrow \infty} A\left(x_{n}\right)=\lim _{n \rightarrow \infty}\left(5+\frac{1}{n}\right)=2, \\
& \lim _{n \rightarrow \infty} S\left(x_{n}\right)=\lim _{n \rightarrow \infty}\left(5+\frac{1}{n}\right)=2,
\end{aligned}
$$

and

$$
\begin{aligned}
& \lim _{n \rightarrow \infty} S A\left(x_{n}\right)=S(2)=2, \lim _{n \rightarrow \infty} A A\left(x_{n}\right)=2=A(2), \\
& \lim _{n \rightarrow \infty} A S\left(x_{n}\right)=\frac{8}{3} \neq 2=A(2),
\end{aligned}
$$

which shows that the pair $(A, S)$ is subsequently continuous of type $(A g)$ as well as $R$ weakly commuting of type (Ag) but not reciprocally continuous and compatible. Moreover, one can also check inequality (2.3) by defining $F$ as $F\left(t_{1}, t_{2}, t_{3}, t_{4}, t_{5}, t_{6}\right): \mathbb{R}_{+}^{6} \rightarrow \mathbb{R}$ as

$$
F\left(t_{1}, t_{2}, t_{3}, t_{4}, t_{5}, t_{6}\right)=t_{1}-k\left(\max \left\{t_{2}, t_{3}, t_{4}, t_{5}, t_{6}\right\}\right)
$$

where $k \in\left[\frac{1}{2}, 1\right)$. Thus, all the conditions of Corollary 2.2 are satisfied. Notice that 2 is a coincidence as well as unique common fixed point of the pair $(A, S)$.

This example cannot be covered by those fixed point theorems which require both compatibility and reciprocal continuity or completeness (or closedness) of the underlying spaces or subspaces. Notice that in this example neither $X$ is complete nor $A(X)=\{2\} \cup\left(\frac{8}{3}, \frac{20}{3}\right]$ or $S(X)=[2,7) \cup\{18\}$ is closed (e.g., $\left.[3,13,18]\right)$. 


\section{Author details}

${ }^{1}$ Department of Mathematics and Humanities, S.V. National Institute of Technology, Surat, Gujarat, India ${ }^{2}$ Department of Mathematics, Aligarh Muslim University, Aligarh 202 002, India ${ }^{3}$ Department of Mathematics, Lahore University of Management Sciences, Lahore 54792, Pakistan

\section{Authors' contributions}

All authors carried out the proof. All authors conceived of the study, and participated in its design and coordination. All authors read and approved the final manuscript

\section{Competing interests}

The authors declare that they have no competing interests.

Received: 19 April 2011 Accepted: 20 February 2012 Published: 20 February 2012

\section{References}

1. Babu, GVR, Alemayehu, GN: Common fixed point theorems for occasionally weakly compatible maps satisfying property (E.A.) using an inequality involving quadratic terms. Appl Math Lett. 24, 975-981 (2011). doi:10.1016/j.aml.2011.01.008

2. Kameswari, MVR: Existence of Common fixed points, equivalence of the convergence of certain iterations and approximations of fixed points of multi-valued maps. Andhra University, India (2008) Doctoral Thesis

3. Ali, J, Imdad, M: An implicit function implies several contraction conditions. Sarajevo J Math. 17(4):269-285 (2008)

4. Gopal, D, Imdad, M, Vetro, C: Common fixed point theorems for mappings satisfying common property (E.A.) in symmetric spaces. Filomat. 25(2):59-78 (2011). doi:10.2298/FIL1102059G

5. Aliouche, A: A common fixed point theorem for weakly compatible mappings in symmetric spaces satisfying a contractive condition of integral type. J Math Anal Appl. 322(2):796-802 (2006). doi:10.1016/j.jmaa.2005.09.068

6. Galvin, F, Shore, SD: Completeness in semi-metric spaces. Pacific J Math. 113(1):67-75 (1984)

7. Hicks, TL, Rhoades, BE: Fixed point theory in symmetric spaces with applications to probabilistic spaces. Nonlinear Anal. 36, 331-344 (1999). doi:10.1016/50362-546X(98)00002-9

8. Wilson, WA: On semi-metric spaces. Am J Math. 53, 361-373 (1931). doi:10.2307/2370790

9. Cho, SH, Lee, GY, Bae, JS: On coincidence and fixed-point theorems in symmetric spaces. Fixed Point Theory Appl 9 (2008). Volume Art. ID 562130

10. Dori'c, D, Kadelburg, Z, Radenović, S: A note on occasionally weakly compatible mappings and common fixed points. Fixed Point Theory (in press). http://www.math.ubbcluj.ro

11. Jungck, G, Rhoades, BE: Fixed point theorems for occasionally weakly compatible mappings. Fixed Point Theory 7(2):287-296 (2006)

12. Jungck, G, Rhoades, BE: Erratum to fixed point theorems for occasionally weakly compatible mappings. Fixed Point Theory. 7(2):287-296 (2006)

13. Pathak, HK, Cho, YJ, Kang, MS: Remarks on R-weakly commuting mappings and common fixed point theorems. Bull Korean Math Soc. 34(2):247-257 (1997)

14. Bouhadjera, H, Godet-Thobie, C: Common fixed point theorem for pair of subcompat-ible maps. (2009) arXiv:0906.315901[Math.FA]

15. Abbas, M, Gopal, D, Radenovi'c, S: A note on recently introduced commutative conditions (submitted).

16. Ali Alghamdi, Mohammad, Radenovi'c, Stojan, Naseer, Shahzad: On some generalizations of commuting mappings. Abst and Appl Anal 6 (2012). Volume Article ID 952052

17. Imdad, M, Ali, J: Tanveer, M: Remarks on recent metrical fixed point theorems. Appl Math lett. 24, 1165-1169 (2011). doi:10.1016/.j.aml.2011.01.045

18. Pant, RP: Common fixed points for four mappings. Bull Calcutta Math Soc. 9, 281-286 (1998)

doi:10.1186/1687-1812-2012-18

Cite this article as: Gopal et al: Metrical common fixed point theorems without completeness and closedness.

Fixed Point Theory and Applications 2012 2012:18.

\section{Submit your manuscript to a SpringerOpen ${ }^{\odot}$ journal and benefit from:}

- Convenient online submission

- Rigorous peer review

- Immediate publication on acceptance

- Open access: articles freely available online

- High visibility within the field

- Retaining the copyright to your article

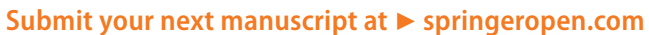

